\title{
Highly Sensitive Centrality Dependence of Elliptic Flow - A Novel Signature of the Phase Transition in QCD
}

\author{
H. Sorge \\ Department of Physics \& Astronomy, SUNY at Stony Brook, NY 11794, USA
}

(December 21st, 1998)

\begin{abstract}
Elliptic flow of the hot, dense system which has been created in nucleus-nucleus collisions develops as a response to the initial azimuthal asymmetry of the reaction region. Here it is suggested that the magnitude of this response shows a "kinky" dependence on the centrality of collisions for which the system passes through a first-order or rapid transition between quark-gluon plasma and hadronic matter. We have studied the system $\mathrm{Pb}(158 \mathrm{AGeV})$ on $\mathrm{Pb}$ employing a recent version of the transport theoretical approach RQMD and find the conjecture confirmed. The novel phase transition signature may be observable in present and forthcoming experiments at CERN-SPS and at RHIC, the BNL collider.
\end{abstract}

One of the most important goals of the heavy-ion programs in the ultrarelativistic energy domain is the search for the phase transition between hadronic matter and the quark-gluon plasma (QGP). After a decade-long effort based on numerous experiments at fixed-target machines (CERN-SPS, BNL-AGS) heavy-ion physics can be considered a mature field today. Thus it may seem surprising that there still is a shortage of reliable signatures for the elusive state QGP and the transition itself [1]. It may be easier to comprehend the difficulties to identify the QGP if one takes into account that properties of the QGP and the transition have to be reconstructed from the final state which obviously is of hadronic nature. On a more fundamental level, it has become clear only recently that the properties of strongly interacting matter even far above the critical temperature $T_{c}$ are essentially non-perturbative. This makes many of the "first-generation" QGP signals which are based on perturbation theory unreliable at best.

On the other side, information about the QGP and the phase transition region has become available with the advent of more powerful lattice gauge simulations of quantum chromodynamics (QCD) [2,3]. Most notably, it has been shown that chiral symmetry is restored at rather low temperatures (in the range 140 to $170 \mathrm{MeV}$ ). Furthermore, the Equation of State (EOS) varies rather rapidly in the transition region. It is not clear yet whether the transition is of weak first-order or just a rapid cross-over between the two phases. The EOS extracted from the lattice clearly displays the transition from hadron to quarkgluon degrees of freedoms. Pressure and energy density approach the ideal Stefan-Boltzmann values at temperatures $\geq 3 T_{c}$. A generic feature of the EOS in the transi- tion region is the presence of the so-called "softest point of the EOS" [4] related to the effect that the energy density may jump with increasing temperature but the pressure does not.

The collective transverse flow which develops in the heavy-ion collisions reflects on the properties of the EOS. Usually, one distinguishes various types of transverse flow, the radial (isotropic component), directed (sideward kick in the reaction plane) and the elliptic flow, the latter being a preferential emission either along the impact parameter axis or out of the reaction plane (squeeze-out) [6]. The general idea why a phase transition may show up in flow observables is rather straightforward. At densities around the softest point the force driving the matter expansion gets weakened. A long time ago, van Hove has suggested that the multiplicity dependence of average transverse momenta may display a plateau and a second rise [7]. So far, it has not been possible to deduce the presence of a phase transition from the transverse momentum spectra. Some time ago we have suggested that the elliptic flow may be a better-suited observable to identify a first-order type phase transition [8]. Here we make good on this promise and present a novel signature of the QCD phase transition. We predict a rather characteristic centrality dependence of the elliptic flow if the created system passes through the softest region of the EOS in the heavy-ion reactions.

Elliptic flow in the central region of ultrarelativistic collisions is driven by the almond-shape of the participant matter in the transverse plane [9]. It was argued in 8 that elliptic flow may be more sensitive to the early pressure than the isotropic radial flow. "Early" and "late" is defined by the time scale set by the initial transverse size $r_{t}=\sqrt{\left\langle x^{2}+y^{2}\right\rangle}$ of the reaction region. Thus early flow appears at times $\approx r_{t} / c$ while we would refer to flow generated at times $>2 r_{t} / c$ as late. One reason for the larger sensitivity of the elliptic flow to early pressure is that the generated flow asymmetry works against its cause and diminishes the spatial asymmetry on a time scale proportional to $\sqrt{\left\langle y^{2}\right\rangle}-\sqrt{\left\langle x^{2}\right\rangle}$. Furthermore, the elliptic asymmetry is proportional to the difference between the flow strengths in $x$ (parallel to impact parameter) and $y$ direction. Thus it is more fragile than radial flow. Viscosity related non-ideal effects tend to wash out the pressuredriven asymmetries. Obviously, these effects will be more pronounced in the later dilute stages of the reaction. Unfortunately, this could not be demonstrated in the earlier work. The transport model RQMD (version 2.3) [10 employed for the calculations lacked any sizable transverse 
pressure in the early stages - a combination of softness from pre-equilibrium motion and absence of a QGP phase which would generate more pressure than the resonance matter simulated in the model. As a result the final hadron momentum spectra showed azimuthal asymmetries much smaller than hydrodynamical results which include a phase transition into the QGP. In the mean time, NA49 has analysed data for semi-central Pb(158AGeV) on $\mathrm{Pb}$ collisions [1]. The measured azimuthal asymmetries are roughly equally distant from the closest results based on hydrodynamics and from the RQMD calculations 12,13. Both of these calculations show a factor of two disagreement, however, in different directions. In this Letter we are going to present results from calculations with a new version of the transport model RQMD (version 3.0) which incorporates an EOS with 1st order phase transition. Comparing to the results obtained in the model without QGP phase we may assess the importance of the phase transition.

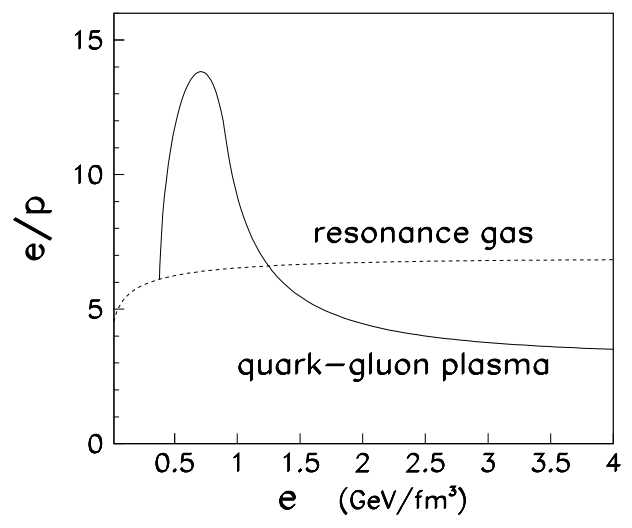

FIG. 1. Equation of states implemented into RQMD: ratio of energy density $e$ divided by pressure $p$. The dashed line represents the resonance gas EOS, the solid line the EOS including a first order phase transition with $T_{c}=160 \mathrm{MeV}$.

Let us first shortly describe how the phase transition is implemented into the model. A detailed description of the algorithm will be presented elsewhere. In RQMD nucleus-nucleus collisions are calculated in a Monte Carlo type fashion. While the nucleons from each colliding nuclei pass through each other, they are decomposed into constituent quarks. Strings may be excited, and overlapping strings fuse into ropes (with larger chromoelectric field strength). After their decay and fragmentation secondaries emerge and may interact with each other. Formed resonances are treated as unstable quasiparticles. This leads to a resonance gas EOS if there are no corrections from other interactions. The QCD dynamics in the phase transition region is not well understood. Even if there is a quasi-particle description it is not obvious which one of the many possible choices (strings, constituent quarks, partons, either massless or with dy- namical masses) is to be prefered. In this situation we have decided to stick to the implemented degrees of freedom and modify the collision term instead. Since we expect that hydrodynamics is a reasonable approach for the transverse dynamics in the ultradense stage, the EOS should be the most relevant ingredient for the expansion dynamics anyway. It is well-known that different treatment of interactions between quasi-particles may modify the EOS. In general, if particles are free between interaction points the virial theorem specifies that the pressure of the system in equilibrium is given by 14

$$
P=P_{i d}+\frac{1}{d \cdot V \cdot \Delta T} \sum_{(a, b)}\left(\delta \vec{p}_{a} \cdot \vec{r}_{a}+\delta \vec{p}_{b} \cdot \vec{r}_{b}\right)
$$

The first term arises from free streaming. The second term represents the non-ideal contribution $\Delta P$ due to momentum changes $\delta \vec{p}_{a}$ at discrete collision points $\vec{r}_{a}$. $d=3$ is the number of space dimensions, $V$ the volume of the system, $\Delta T$ a sufficently large time interval, and the summation goes over all collisions. $a$ and $b$ specifies which quasi-particles collide. The standard collision term in RQMD is manufactured such that it does not contribute to the pressure. Now, we depart from this "ideal" collision term and let each quasi-particle interact elastically with a neighbor after any of the standard collisions. The momentum change is constrained by

$$
\left\langle\delta \vec{p}_{a} \vec{r}_{a}\right\rangle+\left\langle\delta \vec{p}_{b} \vec{r}_{b}\right\rangle \stackrel{!}{=} d \cdot \frac{\Delta P}{\rho} \cdot\left(\Delta t_{a}^{s c}+\Delta t_{b}^{s c}\right)
$$

$\Delta t_{a}^{s c}$ refers to the time which has elapsed since the last of the EOS modulating collisions. $\rho$ is the density of quasiparticles. Introducing collisions according to eq. 0.2 changes the pressure of the system to $P_{i d}+\Delta P$. Eq. (0.2) provides a numerically rather efficient method to modify the ideal EOS. The physics content of eq. (0.2) is that the momentum transfer may be chosen to be either suitably repulsive (QGP at high temperature) or attractive (mixed phase). Fig. 1 displays the ideal EOS based on counting the propagating quasi-particles in RQMD. In addition, an EOS is shown which may be produced by introducing energy density dependent additional interactions according to eq. (0.2). This EOS is the one which will be used for the calculations presented in this Letter. It is calculated from a quasi-particle model of quarks and gluons with dynamical thermal masses [15,16. We have chosen this EOS, because it provides a good fit to lattice data. The EOS contains a 1st order transition at $T_{c}=160 \mathrm{MeV}$ with a latent heat of $467 \mathrm{MeV} / \mathrm{fm}^{3}$. For the RQMD calculations of nucleus-nucleus collisions the novel interaction term is introduced in a local density approximation, i.e. all variables in eq. (0.2) are evaluated in the local rest system of the energy current. Neither is the modulation of the local pressure tensor restricted to regions of local equilibrium nor is - the other extreme - any local equilibration enforced, e.g. by randomizing directions of local momenta. 

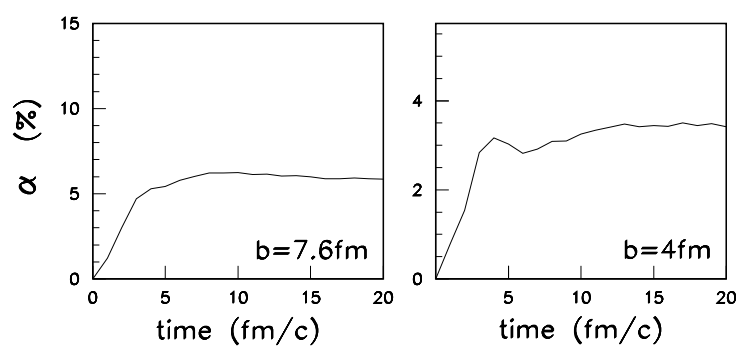

FIG. 2. Time evolution of transverse momentum anisotropy parameter $\alpha$ calculated with RQMD for the system $\mathrm{Pb}(158 \mathrm{AGeV})$ on $\mathrm{Pb}$ at two impact parameters. Only quasi-particles within a central rapidity cut $( \pm 0.7)$ are included.

Let us now turn to results of RQMD calculations which contain a phase transition. We have chosen to do the calculation for the system $\mathrm{Pb}(158 \mathrm{AGeV})$ on $\mathrm{Pb}$, i.e. the heavy-ion reaction at highest beam energy which is currently available. This may be a good place to look for the phase transition. The time evolution of the azimuthal asymmetry parameter $\alpha$ (momentum flow asymmetry)

$$
\alpha=\left(\left\langle p_{x}^{2}\right\rangle-\left\langle p_{y}^{2}\right\rangle\right) /\left(\left\langle p_{x}^{2}\right\rangle+\left\langle p_{y}^{2}\right\rangle\right)
$$

for quasi-particles around midrapidity is displayed in fig. 2. It shows a very different behaviour than corresponding calculations based on RQMD without QGPtype EOS [8]. We see from the figure that in case of QGP formation essentially all of the finally observable asymmetry is created at times smaller than $4 \mathrm{fm} / \mathrm{c}$. The mixed phase leads to a marked dip of the asymmetry for more central collisions. Since the pressure is comparably low, free motion between interactions is able to destroy some of the earlier created flow asymmetry. On the other side, the calculations for semi-peripheral collisions (e.g. $\mathrm{b}=7.6 \mathrm{fm}$ ) show that the softening in the mixed phase cannot stall the expansion of the system. Needless to say that this is a function of the latent heat which is very moderate for the chosen EOS. The overall effect of mixed phase and purely hadronic stage is rather small in a broad impact parameter range. Under the condition of an already well-developed flow asymmetry, diffusive processes and thermal pressure driven work seem to neutralize each other at the later stages. In the QGP-scenario the azimuthal asymmetry is indeed mostly a signature of the early pressure. It is amuzing that non-ideal effects from viscosity in the low-density stage may be helpful to infer information about the pressure in the high-density region.

In the following we will present the main result of the Letter, the measurable azimuthal asymmetry of final hadrons which the experimentalists usually take to be the number flow asymmetry $v_{2}$

$$
v_{2}=\langle\cos (2 \phi)\rangle
$$

as a function of centrality. Tight impact parameter cuts can be obtained using a forward-energy trigger like NA49 has. Of course, the spatial asymmetry of the reaction zone which is correlated with the asymmetry of the participant nucleons in the ingoing nuclei

$$
\alpha_{x}=\left(\left\langle y^{2}\right\rangle-\left\langle x^{2}\right\rangle\right) /\left(\left\langle x^{2}\right\rangle+\left\langle y^{2}\right\rangle\right) \quad .
$$

is itself a function of the impact parameter. Trivially, $v_{2}$ goes to zero for very small and very large impact parameters. The value for $v_{2}$ at any given centrality reflects both the strength of the spatial asymmetry and the response of the created system due to the generated pressure. However, we may disentangle the effects from geometry and dynamics. In general, the final flow asymmetry $v_{2}$ can be viewed as a function of many variables, $\alpha_{x}$, the average initial energy density $e_{0}$, the transverse size $r_{t}$, to name just a few:

$$
v_{2}=f\left(\alpha_{x}, e_{0}, r_{t}, \ldots\right) \approx A_{2}\left(\overline{\alpha_{x}}\right) \cdot \alpha_{x}+\mathcal{O}\left(\left(\alpha_{x}-\overline{\alpha_{x}}\right)^{2}\right)
$$

where we have obtained the second equation from a Taylor expansion around some intermediate value $\overline{\alpha_{x}}$ and taking into account that $v_{2}$ vanishes for $\alpha_{x} \rightarrow 0$. In $\mathrm{Pb}$ on $\mathrm{Pb}$ collisions, $\alpha_{x}$ varies between 0 and 0.50 for impact parameters less than $12 \mathrm{fm}$. Picking an intermediate value of $\overline{\alpha_{x}}$ means that the neglected higher order terms in $\left(\alpha_{x}-\overline{\alpha_{x}}\right)$ are expected to be rather small in practice, on the order of 10 percent. Defining the scaled flow asymmetry as

$$
A_{2}=v_{2} / \alpha_{x}
$$

will therefore allow to assess the dynamical response of the created system to the initial spatial asymmetry.

We display the scaled flow asymmetry $A_{2}$ versus impact parameter $b$ in fig. 3 . Of course, the asymmetry factor $A_{2}$ will tend to vanish in the most peripheral collisions $\left(b \approx 2 R_{P b}\right)$. Initial energy densities are too small, and the system size does not sustain extended reaction times. Both for pions and for protons, $A_{2}$ shows a pronounced variation for smaller $b$ values. This is a result of the EOS softness at intermediate energy densities. However, non-equilibrium effects, in particular partial thermalization initially and system-size dependent freeze-out, also play a major role. Extracted $A_{2}$ values from hydrodynamic calculations [9] show essentially no centrality dependence, except for the grazing collisions. This feature is in marked contrast to the transport calculation which includes the non-equilibrium aspects of the dynamics.

Without phase transition, the asymmetry factor $A_{2}$ calculated from RQMD would simply increase monotoneously with centrality - approximately linearly with the initial system size in the reaction plane $\left(\sim 2 R_{P b}-b\right)$. Indeed, the hard QGP stage of the reaction leads to a rapid increase of the asymmetry $A_{2}$ in collisions with $b \geq 10 \mathrm{fm}$ as is visible from fig. 3 . In this range of centralities the 
initial source size $\sqrt{\left\langle x^{2}\right\rangle}$ along the impact parameter axis is small enough that the associated characteristic time for the development of flow falls within the life time of the QGP phase. In somewhat more central collisions further increase of the asymmetry is cut-off after the system enters into the stage of soft and lateron viscous expansion. Initial energy densities change less with increasing centrality than the system size. Therefore at the characteristic time for flow development typical energy densities are in the region of the softest point. In these reactions increasing reaction time which is helpful to develop the asymmetry is counteracted by the softness of the matter. In any case, the centrality dependence of the flow asymmetry follows a different slope than for the class of more peripheral collisions. For collisions with $b<5 \mathrm{fm}$ kinetic equilibration which takes place on a scale of 3-4 $\mathrm{fm} / \mathrm{c}$ may be realized already in the QGP phase. This gives rise to yet another centrality dependence of the flow asymmetry $A_{2}$ (a second rise).

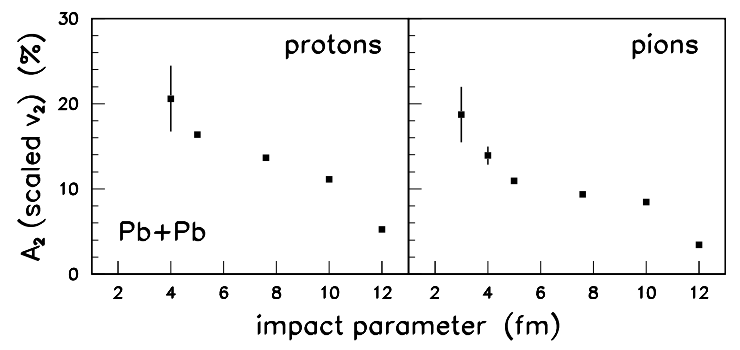

FIG. 3. Scaled azimuthal asymmetry parameter $A_{2}=v_{2} / \alpha_{x}$ of protons and pions as a function of impact parameter for the same system and acceptance window as in fig. 2 .

van Hove's original idea to look for a plateau and a second rise in momentum spectra as a signal of the QCD phase transition may turn out to be true, after all. Present experience tells that it will probably not been found in the multiplicity dependence of average transverse momenta. He did not take into account that the dynamics of the hadronic stages may add a late radial flow component [17] which spoils the original idea. However, if the presented calculations contain some truth it is much better justified to neglect the late hadronic stages for the azimuthal asymmetries of particle spectra. The presented calculation contains some uncertainties. The equation of state is not well-determined in the transition region. The admixture of baryons in the central region and the strong pre-equilibrium deformation of the local stress tensor add to the uncertainties. Nevertheless, the potential rewards in terms of insight into the phase transition dynamics should justify a careful search for structure in the centrality dependence of elliptic flow at SPS and future RHIC energy.

This work has been supported by DOE grant No. DEFG02-88ER40388. After completion of the manuscript the author became aware of a recent work [18] in which the influence of a 1st order transition on elliptic flow is also being discussed.

[1] S.A. Bass, M. Gyulassy, H. Stöcker, W. Greiner: hep$\mathrm{ph} / 9810281$.

[2] G. Boyd, J. Engels, F. Karsch, E. Laermann, C. Legeland, M. Lütgemeier, B. Petersson: Nucl. Phys. B469 (1996) 419.

[3] J. Engels, R. Joswig, F. Karsch, E. Laermann, M. Lütgemeier, B. Petersson: Phys. Lett. B396 (1997) 210.

[4] C.M. Hung, E.V. Shuryak: Phys. Rev. Lett. 75 (1995) 4003.

[5] D.H. Rischke, Y. Pursun, J.A. Maruhn: Nucl. Phys. A 595 (1995) 383.

[6] A.M. Poskanzer, S.A. Voloshin: Phys. Rev. C58 (1998) 1671.

[7] L. van Hove: Phys. Lett. B118 (1982) 138.

[8] H. Sorge: Phys. Lett. B402 (1997) 251.

[9] J.Y. Ollitrault: Phys. Rev. D46 (1992) 229; Phys. Rev. D48 (1993) 1132.

[10] H. Sorge: Phys. Rev. C52 (1995) 3291.

[11] H. Appelshäuser et al. (NA49): Phys. Rev. Lett. 80 (1998) 4136.

[12] J.Y. Ollitrault: Proceedings of Quark Matter '97, Nucl. Phys. A in print.

[13] H. Liu, S. Panitkin, N. Xu: nucl-th/980721, Phys. Rev. $\mathrm{C}$ in print.

[14] P. Danielewicz, S. Pratt: Phys. Rev. C53 (1996) 249.

[15] A. Peshier, B. Kämpfer, O. P. Pavlenko, G. Soff: Phys. Rev. D54 (1996) 2399.

[16] B. Kämpfer, O. P. Pavlenko, A. Peshier, M. Hentschel, G. Soff: J. Phys. G23 (1997) 2001.

[17] H. van Hecke, H. Sorge, N. Xu: nucl-th/9804035, Phys. Rev. Lett. in print.

[18] H. Heiselberg, A. Levy: nucl-th/9812034. 